International Journal of Engineering \& Technology, $7(2.7)(2018) 746-753$
International Journal of Engineering \& Technology
SPC
Website: www.sciencepubco.com/index.php/IJET
Research Paper

\title{
A Comparative analysis of the methods used for building Information / Content Centric Networks over Software defined networks
}

\author{
B.Vishnu Priya ${ }^{1}$, Dr. JKR Sastry ${ }^{2}$ \\ ${ }^{1}$ Department of computer science and engineering, KLEF deemed to be University, vaddeswaram, \\ Guntur District, Andhra Pradesh, India \\ ${ }^{2}$ Department of computer science and engineering, KLEF deemed to be University, vaddeswaram, \\ Guntur District, Andhra Pradesh, India \\ *Email: vishnupriya574@gmail.com
}

\begin{abstract}
Ability to transfer huge amount of content to the target is the present-day requirements of the users which is not being used through internet-based protocol due to static nature of the internet. Software defined networks (SDN) provides the flexibility to implement any architecture as the control and data plane are separated. Information / content centric networks (ICN / CCN) can be implemented using SDN. The requirement of the massive delivery of the content can be archived through ICN/CCN.In this paper a comparative analysis of the methods used for building information centric networking ICN / CCN over software defined networks has been presented. The areas of research that needs to be undertaken further have also been cited in the paper.
\end{abstract}

Keywords: Content centric networks (CCN); Internet protocol (IP);Information centric Networks (ICN); Massive content delivery; OpenFlow Protocol; Software defined networks (SDN).

\section{Introduction}

The advent of internet has brought in radicals in the way information is communicated. The invention of WEB technologies has revolutionized the way the data is disseminated. However, the data the way it communicated over the internet is more about transmitting over the medium, the channels, channel managements etc. The routing of the data more or less is dependent on the IP addresses. The band width of the channels generally is wasted due to the varying nature of the data that must be transmitted over the networks.

Many heterogeneous networks have been invented for effecting communication which includes integration of Wi-Fi with TCP/IP, Cellular with TCP/IP etc. Even in the case of heterogeneity communication is affected primarily through TCP/IP.

One of the main concerns is to be able to implement flexible architecture for effecting the communication which requires that the control layer and data layer needs to be separated so that the control layer can be implemented using flexible architectures. This consideration has lead development of software defined network. Through software the control layer is dynamically configures as per the data communication requirements.

Few tools have been developed that help defining the networks to which the software to the networking units can be programmed and attached. OpenFlow is one such tool. Open flow kind of tools will help in developing software defined networks. All networking gadgets connected with the controlling can be loaded with software and therefore can be made to be active as per the execution results of the software.

While that being the case, the implementation of flexible control layer of the network has resulted into the issues related to scalabil- ity, dynamic configurability etc. Routing of the data is the next issue which must be done based on the kind of data, length of the data and recipient of the data. This requirement leads into implementation of information centric networks on top of software defined networks.

Research has gone deep into this area and many contributes have been presented. An overview on the same is required so at to find the extent of investigations that have gone into and the areas which must be investigated further.

This paper presents comparison of various findings made in the implementation of ICN over SDNs and laid focus on the future research directions.

\section{Related work}

Designing networks purely keeping in view the flow of information is the latest trend. The new trend has been of focus to the industrialists and researchers. Information centric networking (ICN) closely follows the way the information is processed over internet.

Open flow is a tool using which networks of different designs can be deployed with ease over the actual networking resources. A specific ICN solution is deployed using OpenFlow tool in two phases using two platforms that include VSwitch, and NetFPGA. The ICN solution presented includes the functionality related to data naming, in-network caching and route-by-name [1].

Software defined networks (SDN) and ICN (Information centric networking) are being considered as the basic solutions for effective content management. SDN can be used to deploy alternate architectures dynamically while the network is up and running. Information centric networking show the network is designed 
looking at the way the information is moved over the network. Combining SDN and ICN will help deploying a networking architecture that is more information centric. Even the issue related to testing the SDN when ICN is deployed is equally important.

ICN is presented in relation to an architectural context defined through SDN. A novel design of an ICN is presented in the context of SDN. Existing networks have been used for testing ICN in the context of SDN [2].

Information-Centric Networking (ICN) is characterized through components which include in-network caching, routing through defined routes, and Content-centric request/reply for distributing the data

A framework called CONET suitable for development of ICN has been presented. A solution based on CONET has been developed which is also named as coCONET. CoCONET follows the philosophy of SDN. The implementation of coCONET on SDN has been presented and has been shown how the characteristics of coCONET help deploying the ICN on SDN. The deployment is undertaken using OpenFlow network which is the most popular solution for deploying SDN. The Modifications to the OpenFlow have been presented clearly showing the way the modified SDN is suitable for ICN based solutions [3].

Information Centric Networking (ICN) is a new paradigm in which the network layer provides users with content, instead of providing communication channels between hosts, and is aware of the name (or identifiers) of the contents.

Network layer provides content to the users instead of communication channels in the case of ICN. The network layer has the knowledge of the content being communicated with the help of identifiers attached to the content.

One of the main issues that must be addressed is the routing of the content request to the node that is capable of providing the content.

A fundamental ICN operation is the routing of content requests towards a node that is capable to provide the requested content Several routing architectures have been presented in the literature.

Several types of networks can be created based on the routing architecture. One such architecture is the one effecting the routing based on the name of the content and routing table that has the key element being the name of the content.

WEB contents can be hosted on an ICN in which case many name-based routes are to be considered due to the existence of huge content and the need to find the Unique names to the contents. In this case the existing IP based routers cannot hold all name-based routes due the limitation of exiting forwarding information based (FIB) technology. It would be costly to select a hardware that can hold Routing Information base $\{$ RIB $\}$. To counter these disadvantages, a routing-by-name architecture has been presented which is called "Lookup-and-cache". In this case FIB is a cache of routes whereas the RIB is stored at a remote and central location. ICN nodes in the network need to handle fewer routes in the FIB even though huge traffic flows across the network.

The Lookup-and-cache solution has been implemented within CCnx software framework. The system performance has been assessed in terms of download delay, lookup rate and fairness [4].

Another approach to managing the traffic in SDN centric networks is to implement metadata driven services by extracting metadata of the content at network layer. This approach leads to content centric architecture shadowed on to SDN. The ability to extract metadata at network layer will help to build number of new services that can run on the network. Some of the applications that can be implemented when metadata is extracted at network layer include the following [5]:
1. Optimization of content delivery based on content length

2. A content firewall built using the metadata. A differentiated treatment can be affected by the firewall based on kind of content that needs to be delivered.

A method of transferring data between a software defined network (SDN) and an information-centric network (ICN), wherein the method comprises receiving a request from an SDN node for a specific named content stored on an ICN, wherein the request is encapsulated in an Internet Protocol (IP) packet, encapsulating the IP packet using an IP protocol stack, parsing the request to obtain the name of the specific named content, finding a path to an ICN networking device hosting the specific named content using the name, and forwarding the packet to the ICN networking device over the path.

A method has been developed that effects transferring data between ICN and SDN. The implementation involves receiving a request from a SDN node for the required content stored in an ICN The request is encapsulated into an IP packet using IP protocol stack. The request is parsed to obtain the name of the content leading to finding the path to ICN networking device that hosts the requested content, and then forwarding the content to the device over a path.

A method has been implemented that obtains metadata from the content requested by a client device, allocating one or more network resources that hosts the data based on the metadata and then sending a message that identify the allocated resources to a switch which then directs the content to be served to the client. The method is implemented in a network controller which controls the switch. The controller configures the switch to forward the content to the client devices through network resources allocated.

Efficient content dissemination can be achieved through $\mathrm{CCN}$ through caching the content on the path from provider to the consumer. This way, the consumer experience and cost is improved. This approach proves to be inefficient within a domain. Caching in $\mathrm{CCN}$ through software defined networking approach can improve the performance [6].

A CNN can be enabled on the existing IP network such as on an ISP or data center through the functions and control implemented by SDN. A mechanism has been presented that does not require either an extended network or additional protocols. The mechanism does not require any changes in ICN host network stacks. The mechanism supports aggregation of routes to be undertaken with SDN controlled network. The ICN protocol in use does not require all the networking elements to be SDN enabled. Advanced ICN routing features such a segregation, forking, load balancing, and traffic engineering explicit path steering through ICN caches. The method is implemented using the open flow controller framework and CCNx [7]

ICN can be implemented on existing IP networks such as ISP or a data Centre network using SDN related functions and controls.

A mechanism has been presented that allow transfer of information through internet, identify the ICN requests and responses, decoupling the contents from its identifiers and forward the packets in the normal IP methods. The method does not require any extension of either the network or protocols and there is no need to make changes to either the client or server operating systems (OS). The method support aggregation of routes inside SDN.

The mechanism is not quite dependent on specific ICN protocol and it is not necessary that all the network elements to be SDN Enabled. Many ICN routing features that include request aggregation, load balancing, forking, explicit path steering through ICN caches, which are advanced, has been included into the method. 
The design of the networking that implements the proposed method has been implemented using Trema Open Flow controller framework and CCNx (Content-Centric Networking - Reference Implementation) [8]

Users are more concerned in moving content between the hosts than the channels that carry the content. ICN is more concerned with delivering the content, SDN on the other hand helps is implementing different kinds of networking architectures as they evolve. Using ICN over SDN helps to implement networks which are content centric and can evolve into different architectures as they evolve.

CONET is a ICN framework that is built on CCNx reference model. The CONET is extended to comply with SDN requirements

An approach has been followed that first implements an SDN which is generic in nature without much worrying about the standards and equipment used to setup a SDN network and then implement ICN within the SDN using OpenFlow framework [9].

One of the main problems of the internet is wastage of bandwidth, excessive peer to peer traffic and millions of content delivery networks. Content centric networks solve the problems that exist when communication is undertaken using internet. Content centric networks $(\mathrm{CCN})$ will help moving way form host centric architecture to content centric architecture.

Many issues have to be addressed when one wants to deploy CCN migrating away from internet. Testing actual working of CCN is also complex as there are not many test beds available.

OpenFlow Framework provides programmable environment while keeping the existing network as it is without any necessity to make changes. Not many $\mathrm{CCN}$ implementations have been presented using OpenFlow. Most of the investigations / studies ended at conceptual designs only. A CCN has been implemented using Open-flow keeping in view of achieving end-to-end communication. The issue of the naming the content has been resolved through generating hash values and also the uniqueness of the names is achieved through longest prefix match. The advantage of dealing with more attributes of CNN such as high-speed forwarding, network slicing, routing, caching and enforcing the security etc. for effective deployment of CCN has been presented [10].

Devices can be connected to a network through which services to the end users can be provided. The network device can be programmed to provide transport and service access which are required for handing context-aware logistics and mobility services overlaid on $\mathrm{CCN}$ transport layer. The method is designed for receiving a message that contains a service request in the transport layer. In the service layer (SAL) contextual information, information related to access network are extracted along with the service identities, the data that is needed to execute the service request. The service identities identify the kind of services that are expected.

Downloading the content is becoming frequent phenomenon even through use of smaller devices such as smart devices, smart phone, pad etc. The use of bandwidth will be very high when down loading the content takes place even with small devices.

Long Term Evolution (LTE) devices are expected to be used extensively making it necessary to manage networks efficiently and also controlling the content delivery efficiently in the mobile environment.

A framework has been proposed for establishing SDN based network that support content delivery, network management and network control using LTE devices. A Set of functions have been developed that are related to message forwarding, and SDN switching and built into network entities such as Packet data Gateway (P-GW), Service-Gateway (S-GW) and evolved Node B (eNB). The functions are developed considering efficient content delivery and network management. The SDN controller function has also be developed and built into Mobility Management Entity (MME) [11].

There has been extensive demand for moving multimedia-based content over internet. Information centric networks (ICN) are being evolved extensively so that media content is transported at least significant cost. Several ICN architectures are in vogue. Optimal Use of In-network cache has been the key component in ICN

The optimal us of In-network cache requires the consideration of appropriate caching strategy, content routing and linear network coding. A cache management framework has been proposed for ICN networks based on SDNs wherein the controller is programmed for determining the optimal caching strategy and content routing through linear network coding. The framework is based on minimizing the required bandwidth for content delivery. An algorithm for cache management based on network coding has been developed that helps to arrive at near optimal caching and routing in ICNs [12].

Quite recently effort is on hand for integrating SDN and ICN with the main aim of efficient content delivery with minimum bandwidth. SDN as such deals with two planes that include control plane and data plane. Scaling the control plane within SDN as required might become complicated with SDN-ICN networking due to two new features added which include in-network cache and content-based communication. A scalable area based hierarchal architecture (SAHA) has been presented for deciding on the location of controller for deployment. In SAHA, the scaling up network resources that have built-in awareness of the network, content resources are considered which guarantees the efficient matching and resource adaption [13].

One of the most important features of ICN is in-network caching. The In-network caching can reduce the network traffic, remove server bottlenecks, and reduce network latencies. Many innetwork caching methods have been presented in the past that aim at improving hit ratio and cache hit distance. A survey has been presented that provides comprehensive overview of the all the innetwork caching algorithms bring out the advantages and drawbacks [14].

The name-based routing which is the core of an ICN allows the users to ask for data by name and the infrastructure with ICN delivers the content to the users through nearby cache if available.

Low cost and low complexity of network management can be achieved through implementing SDN which is achieved through separating the infrastructure and architecture. While data flow is managed through infrastructure the control flow is achieved through the architecture. This kind of strategy helps continuous evolution of architecture without much bothering about infrastructure.

The combination of SDN that provides efficient network management and ICN that aims at efficient dissemination of the data helps in implementing a framework that lead to efficient data dissemination. A ICN architecture within the framework of SDN has been presented [15].

The demand for Content based web services is increasing these days. The end-to-end model supported by internet has been proved to be in-effective when it comes to effecting content-based delivery. ICN has been in implementation to address the content-based delivery. A survey is presented on the current state of ICN consid- 
ering the key aspects of ICN which includes in-network caching, routing schemes, and content naming [16].

$\mathrm{P} 2 \mathrm{P}$ and video streaming forms the main part internet traffic. Internet is found to be infective to handle such traffic and as a result $\mathrm{CCN}$ which is content centric has been introduced. The deployment of CCN in large scale is still the problem due to lack of scalable routing algorithm.

Content delivery such as $\mathrm{P} 2 \mathrm{P}$ or video streaming generates the main part of the Internet traffic and Content Centric Network $(\mathrm{CCN})$ appears as an appropriate architecture to satisfy the user needs.

SDN is used for separating data and control planes and a new routing scheme called SRSC has been proposed to be implemented within CCN. Only CCN messages and the SDN is used for content delivery.

In this paper we propose to use the Software-Defined Networking (SDN) paradigm to decouple data plane and control plane and present SRSC, a new routing scheme for $\mathrm{CCN}$. The numbers of messages required for communicating have been reduced while at the same time improving the performance of the Cache within CCN [17].

OpenFlow is a framework that can be used for defining SDN and $\mathrm{CCN}$ networks. OpenFlow supports a set of protocols that are used for effecting the communication over the network. Enabling an ICN through use of SDN functions which can be activated through an SDN instantiation can be achieved through a OpenFlow protocol function.

CCNx a virtual ICN network, Vswitch (A virtual switch in SDN), Floodlight a kind of controller in SDN are used for designing a network. A test bed has been developed that can accommodate routing process of ICN in SDN, identify the content requests, effect efficient content delivery, and also enable the existing IP networks has been built and presented. The model presented supports the tradition host-to-host communication using TCP/IP. The way Vswitch should be altered for matching the operations of $\mathbf{C C N x}$ has been presented [18]

Most of the networks these days are heterogeneous (HetNet) in especially combining wireless with TCP/IP, wireless with cellular etc. Many services are being provided through millions of applications through several content providers and network service providers. The communication is driven through host centric protocol such as TCP/IP. ICN networks are information centric. Information dissemination is more important than the way the information is communicated over the network. Network traffic is dependent on the way data is dominated rather than the way is communicated. It is necessary that all the network entities must coverage to take the advantage of smart traffic transmissions, scalability and openness.

A new radio network is merging that forms into a cloud which is aimed at developing the infrastructure and network management in its simplest manner. The networking is called C-RAN. An attempt is being made to integrate ICN protocol with C-RAN architecture for achieving efficient information management and communication.

On the other hand, SDNs have come up that help re-configuring the HetNets which can be controlled through wired networks. The ICNs, C-RANs and SDNs are complimentary to each other's in the way the all the architectures can be integrated within a het-net [19].

High volume of vital information related vehicle movement or vehicle safety gets transmitted across the network that connects the vehicles. The data is exchanged between vehicle-built transmitters ad road side receiving and transmitting systems. The relia- bility of the data delivery will be generally poor due to weak wireless links and mobility of the vehicles. The vehicular system as such is dynamic which can be served through a static IP centric networking system. Location specific communication systems cannot be addressed by IP centric networking systems.

The challenging and ever evolving dynamic requirements of vehicular networks can be met by through implementing information centric system. The features of ICN that include named content, support for multicasting, in-network caching greatly helps in implementing vehicular networks. Much architecture are in use for implementing vehicular systems. Interoperability between these architectures is needed when a comprehensive, composite and complete network is needed. The application networks need to be implemented using the latest networking paradigms such as ICN, CCN and SDN [20]

The satellite and terrestrial networks are undergoing see change with increase in $5 \mathrm{G}$ mobile wireless requirements, mobility support, flexibility reaction and integration. All these aspects are dynamic in nature and therefore cannot be addressed using static networks. ICN and SDN being dynamic can address these problems. An ICN architecture based on ICN has been proposed for implementing satellite terrestrial integration network so as to achieve seamless integration and provide high quality of communication services [21]

Information-Centric Networking (ICN) is being used as a standard for efficient content delivery and SDN is being used for providing efficient management of the network. Use of both ICN and SDN provides highly controllable network coupled efficient data delivery.

A network is designed based on CCnx which a reference model for is implementing ICN, VSwitch as a virtual SDN switch, Floodlight as SDN controller and a wrapper that helps VSwitch to access ICN named data packets.

The content delivery through such a network is found to be much efficient compared to traditional network in terms of content delivery, higher throughput, less packet loss and jitter [22].

Users can get data by inputting a named object and the ICN network will deliver the content from a nearby cache when innetworking cache is implemented. ICN implements the namebased routing.

Efficient network controlling, and management can be implemented by separating data layer and control layer which is achieved through Software-Defined Networking (SDN). Using SDN flexible networking environment is provided.

ICN can be deployed over SDN due to the flexibility built into SDN. A design has been presented that implements ICN over SDN which provides the following:

i) Efficient content delivery with very less transmission delay

ii) Users can request for data using the data names

iii) Higher through put in terms of number of packets delivered per second

iv) Negligible packet loss and jitter

The design is implemented through Open Flow protocol and no modification has been made to the protocol ensuring that IP can still be used [23].

P2P file sharing and video streaming involves major chunk of data transmission over the internet. Internet uses IP as a protocol for delivery which cannot handle large scale delivery of the content. ICN and in particular the NDN is an excellent architecture that can 
cater to delivering content on large scale. NDN does not use routing protocol and data is transmitted through flooding. Both SDN and ICN can be used together and a routing strategy implemented through SDN controller. A cache replacement policy has been based on statistical analysis is implemented in the switch which is used for network management based on the kind of controlling that must be undertaken [24].

\section{Comparative Analysis}

The contributions presented in the literature, over of which has been presented in section 2. The comparison has been made considering tools and framework used, the constituent elements of SDN, the features of ICN used for building information centric networks, and the parameters that were used for comparing the effectiveness of the networks vis-à-vis traditional and conventional IP based networks. The comparison is shown in Table 1 .

OpenFlow tool is generally used for building the networks added with programming facility thus providing a facility required for setting software defined networks. Two frame works are in existence that includes CONET and CCNx using which the ICN can be built and the same can be integrated with Openflow using which SDN can be developed, thus paving a way to integrate ICN with SDN.

OpenFlow comes with built in controller and routing system which have been recommended to be changed to incorporate new switching and routing algorithms. VSwitch is a software controller and Flooding gateway is the routing unit built into OpenFlow using which SDN can be built[25-31].

Many improvements have been suggested in literature for enhancing the capability of SDN networks. Several methods have been presented using which rooting of the content can be explained that include flooding, segregation, forking, load balancing, aggregation of the routes, explicit path steering etc.

The controlling function that should be used for controlling the flow of data can also be programmed and built into OpenFlow tool by modifying the VSwitch component of the OpenFlow tool.

Many contributions have been made to bring the issue of names to the data so that user can refer to the data using names leading to routing based on the content names. Some of the contributions include use of metadata in network layer, parsing IP packets, slicing control area so that scalability of a network can be achieved. Several features that are to be considered for affecting the information centric networking have been traced. Some of the implementations that have been considered for affecting the information centric networking include naming data, in-network-caching, route by name, request aggregation, load balancing, forking, explicit path steering, network slicing, and high-speed forwarding.

The effectiveness of implementation of ICN is evaluated as against the conventional IP based networking considering loss of packets, jitter, download time, lookup rate and fairness. Several applications have been implemented and case studies of the same have been presented which include vehicle networking, mobile communication using satellite networking.

Routing strategies and maximization of the content delivery based on the type of data to be communicated such as Audio, Video, Graphics, animations. Discrete, cardinal and continuous data transmissions and the related issues have not been considered as yet.

\section{Conclusion}

Communicating over traditional networks is more related channels, channel optimizations and channel optimization. Routing in such networks is static irrespective of any kind of data and any amount of data to be transmitted. No consideration is given to the type of data being transmitted. Routing is done in fixed way. The TCP/IP protocol per say is not good enough for handling heavy payloads of the data to be communicated. The routing and seed of communication must be depending on the amount of data to be transport- ed and the recipient for such a data. Software defined networks separates the control and data layers. The architectures that can be used for implementing control layers could be dynamic and flexible, Software define networks provides the flexibility required. The control logic per say can be changed.

Routing the data over the network must be content centric. Utmost importance is given to the data that must be transmitted. The routes of communication have to be decided based on the kinds of data and extent of data that can be communicated. The naming of the data is required so that same can be cashed and delivered as and when the same is requested by the user. The large-scale data communication requirements can be met by implementing ICN/CCN based networks on top of SDN network 


\subsection{Figures and tables}

\begin{tabular}{|c|c|c|c|c|c|c|c|c|c|c|c|c|c|c|c|c|c|c|c|c|c|c|c|}
\hline & & & Software def & ned Netw & & & & & & rmati & $\mathrm{Co}$ & & ntric & netw & vorkin & & & & & & & & \\
\hline $\mathrm{f}$ & & & & & & & & & ea & ures $u$ & & & & & & & & Ass & ssed & Par & amete & & \\
\hline $\begin{array}{l}\mathrm{r} \\
\mathrm{e} \\
\mathrm{n} \\
\mathrm{c} \\
\mathrm{e} \\
\mathrm{N} \\
\mathrm{u} \\
\mathrm{m} \\
\mathrm{b} \\
\mathrm{e} \\
\mathrm{r}\end{array}$ & $\begin{array}{l}\text { Typ } \\
\mathrm{e} \text { of } \\
\text { net } \\
\text { wor } \\
\mathrm{k} \\
\text { used }\end{array}$ & $\begin{array}{l}\text { Fra } \\
\text { me } \\
\text { wor } \\
\mathrm{k}\end{array}$ & Controller & $\begin{array}{l}\text { Root- } \\
\text { ing } \\
\text { Meth- } \\
\text { od }\end{array}$ & 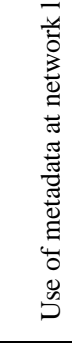 & 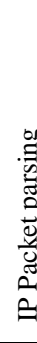 & $\begin{array}{l}9 \\
\vdots \\
0 \\
0 \\
0 \\
0 \\
0 \\
0 \\
0 \\
0\end{array}$ & & & 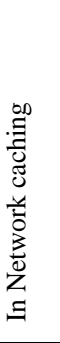 & 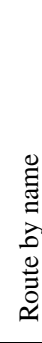 & & 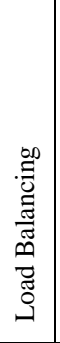 & : & 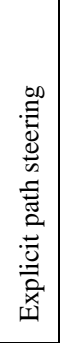 &  & 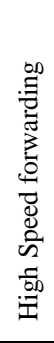 & 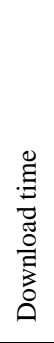 & 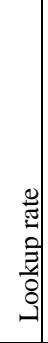 & : &  & : & $\begin{array}{l}\text { Typ } \\
\text { e of } \\
\text { ap- } \\
\text { pli- } \\
\text { cat- } \\
\text { ion }\end{array}$ \\
\hline 1 & $\begin{array}{l}\text { Ope } \\
\text { nflo } \\
\text { w }\end{array}$ & - & VSwitch & $\begin{array}{l}\text { Flood- } \\
\text { ing }\end{array}$ & - & $\sqrt{ }$ & - & & & $\begin{array}{l}\text { Reg- } \\
\text { ular } \\
\text { Cach } \\
\text { ing }\end{array}$ & $\sqrt{ }$ & & & & & & & - & - & & & - & \\
\hline 2 & $\begin{array}{l}\text { Ope } \\
\text { nFlo } \\
\text { w }\end{array}$ & - & Native & Native & - & $\sqrt{ }$ & - & & & $\begin{array}{l}\text { Na- } \\
\text { tive }\end{array}$ & $\sqrt{ }$ & & & & & & & - & - & & & - & \\
\hline 3 & $\begin{array}{l}\text { Ope } \\
\mathrm{n} \\
\text { flow }\end{array}$ & $\begin{array}{l}\mathrm{CO} \\
\mathrm{NE} \\
\mathrm{T}\end{array}$ & Native & Native & - & $\sqrt{ }$ & - & & & $\begin{array}{l}\text { Reg- } \\
\text { ular } \\
\text { cach- } \\
\text { ing } \\
\end{array}$ & $\sqrt{ }$ & & & & & & & - & - & & & - & \\
\hline 4 & - & $\begin{array}{l}\mathrm{CC} \\
\mathrm{Nx}\end{array}$ & Native & Native & - & $\sqrt{ }$ & - & & & $\begin{array}{l}\text { Na- } \\
\text { tive }\end{array}$ & $\sqrt{ }$ & & & & & & & $\sqrt{ }$ & $\sqrt{ }$ & & & $\sqrt{ }$ & \\
\hline 5 & - & - & Native & Native & $\sqrt{ }$ & $\sqrt{ }$ & - & & & $\begin{array}{l}\text { Na- } \\
\text { tive }\end{array}$ & $\sqrt{ }$ & & & & & & & & & & & & \\
\hline 6 & - & - & Native & Native & - & $\sqrt{ }$ & - & & & $\begin{array}{l}\text { Na- } \\
\text { tive }\end{array}$ & $\sqrt{ }$ & & & & & & & & & & & & \\
\hline 7 & $\begin{array}{l}\text { Ope } \\
\text { nFlo } \\
\text { w }\end{array}$ & $\begin{array}{l}\mathrm{CC} \\
\mathrm{Nx}\end{array}$ & & $\begin{array}{l}\text { Segre- } \\
\text { gation } \\
\text { Fork- } \\
\text { ing } \\
\text { Load } \\
\text { balanc- } \\
\text { ing } \\
\text { Aggre- } \\
\text { gation } \\
\text { of } \\
\text { routes } \\
\text { Explic- } \\
\text { it path } \\
\text { steer- } \\
\text { ing }\end{array}$ & & $\sqrt{ }$ & - & & & & & & & & & & & & & & & & \\
\hline 8 & - & $\begin{array}{l}\mathrm{CC} \\
\mathrm{Nx}\end{array}$ & $\begin{array}{l}\text { Trema } \\
\text { open flow } \\
\text { controller }\end{array}$ & $\begin{array}{l}\text { Aggre- } \\
\text { gation } \\
\text { of } \\
\text { routes }\end{array}$ & - & $\sqrt{ }$ & - & & & $\begin{array}{l}\text { Na- } \\
\text { tive }\end{array}$ & $\sqrt{ }$ & $\sqrt{ }$ & $\sqrt{ }$ & $\sqrt{ }$ & $\sqrt{ }$ & - & - & - & - & & & - & - \\
\hline 9 & $\begin{array}{l}\text { Ope } \\
\text { nFlo } \\
\text { w }\end{array}$ & $\begin{array}{l}\mathrm{CO} \\
\mathrm{NE} \\
\mathrm{T}\end{array}$ & Native & Native & - & $\sqrt{ }$ & - & & & $\begin{array}{l}\text { Na- } \\
\text { tive }\end{array}$ & $\sqrt{ }$ & - & - & - & - & - & - & - & - & & & - & - \\
\hline 10 & $\begin{array}{l}\text { Ope } \\
\text { nFlo } \\
\text { w }\end{array}$ & - & Native & Native & - & $\sqrt{ }$ & - & & & $\begin{array}{l}\text { Na- } \\
\text { tive }\end{array}$ & $\begin{array}{l}\mathrm{H} \\
\text { as } \\
\mathrm{h} \\
\mathrm{va} \\
\mathrm{lu} \\
\mathrm{e}\end{array}$ & - & - & - & - & $\sqrt{ }$ & $\sqrt{ }$ & - & - & & & - & - \\
\hline 11 & - & - & Native & Native & - & $\sqrt{ }$ & - & & & $\begin{array}{l}\text { Na- } \\
\text { tive }\end{array}$ & $\sqrt{ }$ & & & & & & & & & & & & \\
\hline 12 & - & - & Native & Native & - & $\sqrt{1}$ & - & & & $\begin{array}{l}\text { Na- } \\
\text { tive }\end{array}$ & - & - & - & - & - & - & - & - & - & & & - & - \\
\hline 13 & - & - & Native & Native & - & $\sqrt{ }$ & - & & & $\begin{array}{l}\text { Net- } \\
\text { work } \\
\text { Cod- } \\
\text { ing } \\
\text { De- } \\
\text { ter- } \\
\text { min- } \\
\text { ation } \\
\text { of } \\
\text { cash- } \\
\text { ing } \\
\text { strat- } \\
\text { egy }\end{array}$ & - & - & - & - & - & - & - & - & - & & & - & - \\
\hline
\end{tabular}






Table 1 Comparative Analysis of Building Information / Content Centric Networks over Software Defined Networks

\section{References}

[1] N. Blefari-Melazzi1, A. Detti1, G. Mazza1, G. Morabito2, S. Salsano1, L. Veltri3, An Open Flow-based Testbed for Information Centric Networking, Future Network \& Mobile Summit (FutureNetw), IEEE, 2012.

[2] Dimitris Syrivelis*, George Parisis, Dirk Trossen, Paris Flegkas, Vasilis Sourlas, Thanasis Korakis and Leandros Tassiulas CERTH-ITI, Greece, Pursuing a Software Defined Information-Centric Network, Software Defined Networking (EWSDN), 2012 European Workshop on, IEEE, 2012.

[3] Luca Veltri, Giacomo Morabito, Stefano Salsano, Nicola BlefariMelazzi, Andrea Detti, Supporting Information-Centric Functionality in Software Defined Networks, Communications (ICC), 2012 IEEE International Conference on, ieee, 2012, 10.1109/ICC.2012.6364916.

[4] A. Detti , M. Pomposini, N. Blefari-Melazzi, S. Salsano, Supporting the Web with an information centric network that routes by name, science Elsevier, Elsevier, 2012, http://dx.doi.org/10.1016/j.comnet.2012.08.006 .

[5] Abhishek Chanda, Cedric Westphal, Dipankar Raychaudhuri, Content Based Traffic Engineering in Software Defined Information Centric Networks, Computer Communications Workshops (INFOCOM WKSHPS), 2013 IEEE Conference on, IEEE, 2013, DOI: 10.1109/INFCOMW.2013.6970717.
[6] Xuan Nam Nguyen, Damien Saucez, Thierry Turletti INRIA Sophia Antipolis M'editerran'ee Sophia Antipolis, France, Efficient caching in Content-Centric Networks using Open Flow, INFOCOM, 2013 Proceedings IEEE, IEEE, 2013 , 10.1109/INFCOM.2013.6566716.

[7] Markus Vahlenkamp, Fabian Schneider, Dirk Kutscher, Jan Seedorf, Enabling ICN in IP Networks Using SDN, Network Protocols (ICNP), 2013 21st IEEE International Conference on, ieee, 2013, 10.1109/ICNP.2013.6733634.

[8] Markus Vahlenkamp, Fabian Schneider, Dirk Kutscher, Jan Seedorf, Enabling Information Centric Networking in IP Networks Using SDN, Future Networks and Services (SDN4FNS), 2013 IEEE SDN for, iee, 2013, 10.1109/SDN4FNS.2013.6702539.

[9] S. Salsano, N. Blefari-Melazzi, A. Detti, G. Morabito, L. Veltri, Information centric networking over SDN and Open Flow: $\mathrm{Ar}-$ chitectural aspects and experiments on the OFELIA testbed, Computer Networks, Elsevier Science Direct, 2013, Volume 57, Issue 16, 13 November 2013, Pp:3207-3221.

[10] Atsushi Ooka, Shingo Ata, Toshio Koide, HIDEyuki Shimonishi, and Masayuki Murata, Open Flow-based Content-Centric Networking Architecture and Router Implementation, Future Network and Mobile Summit (FutureNetworkSummit), 2013,IEEE 2013.

[11] Rim Haw, Choong Seon Hong, Sungwon Lee, An Efficient Content Delivery Framework for SDN Based LTE Network, 8th International Conference on Ubiquitous Information Management 
and Communication Article No. 71, ACM, 2014 10.1145/2557977.2558087.

[12] Jin Wang, Jing Ren, Kejie Lu,, Jianping Wang, Shucheng Liu, and Cedric Westphal, An Optimal Cache Management Framework for Information-Centric Networks with Network Coding, Networking Conference, 2014 IFIP, ieee, 2014, : 10.1109/IFIPNetworking.2014.6857127.

[13] Shuai Gao, Yujing Zeng, Hongbin Luo and Hongke Zhang, Scalable Area-based Hierarchical Control Plane for Software Defined Information Centric Networking, Computer Communication and Networks (ICCCN), 2014 23rd International Conference on, IEEE, 2014, 10.1109/ICCCN.2014.6911839.

[14] Meng Zhang, Hongbin Luo, and Hongke Zhang, A Survey of Caching Mechanisms in Information-Centric Networking, IEEE COMMUNICATION SURVEYS \& TUTORIALS, ieee, 2015, VOL. 17, NO. 3.

[15] Suyong Eum, Masahiro Jibiki, Masayuki Muratay, Hitoshi Asaeda, Nozomu Nishinaga, An ICN architecture within the framework of SDN, Ubiquitous and Future Networks (ICUFN), 2015 Seventh International Conference on, ieee, 2015,: 10.1109/ICUFN.2015.7182521.

[16] AthanasiosV. Vasilakon, ZheLi b, GwendalSimon , WeiYou , Information centric network: Research challenges and opportunities, Journal of Network and Computer Applications, Elsevier Science Direct, 2015, vol:52 pp:1-10.

[17] Elian Aubryy, Thomas Silver stonz and Isabelle Chrismenty, SRSC: SDN-based Routing Scheme for CCN, Network Softwarization (NetSoft), 2015 1st IEEE Conference on, IEEE, 2015, 10.1109/NETSOFT.2015.7116130.

[18] Peng Li, Wu Muqing, Wang Ning, and Liu Hongbao, Supporting Information-Centric Networking in SDN, international Journal of Future Computer and Communication, ijfcc, 2015, Vol. 4 No. 6.

[19] Chenchen Yang, Student Member, IEEE, Zhiyong Chen, Member, IEEE, Bin Xia, Senior Member, IEEE, Jiangzhou Wang, Senior Member, IEEE, When ICN Meets C-RAN for HetNets: An SDN Approach, IEEE Com munications Magazine, IEEE, 2015, Volume: 53, Issue: 11, 10.1109/MCOM.2015.7321980.

[20] [20] Marica Amadeo, Claudia Campolo, Antonella Molinaro,Inform

[21] ation-Centric Networking for Connected Vehicles: A Survey and

[22] Future Perspectives, IEEE Communications Magazine, IEEE, 2016 ,

[23] Volume: 54, Issue: 2, 10.1109/MCOM.2016.7402268.

[24] [21] Yuan Zhang, Ying Wang, SDN Based ICN Architecture for the Future Integration Network, Communications and Information Technologies (ISCIT), 2016 16th International Symposium on, IEEE, 2016,: 10.1109/ISCIT.2016.7751677.

[25] [22] A.GOPI, Dr.A. Nagesh, Performance Evaluation for Information Centric Networking over Custom-SDN Network, International Journal of Engineering Research, ijer, 2017, Volume No.6, Issue No.3, pp :178-183.

[26] [23] Mareb H. Atiyah, Dr. Ammar D. Jasim, Supporting Information-Centric Networking in SDN Data Centre Network, Annual conference on new trends in information \& communication technology applications(ntict), IEEE, 2017, 10.1109/NTICT.2017.7976142.

[27] [24] Anwar Kalghoum, Sonia Mettali Gammar, Towards new Information

[28] Centric Networking strategy based on Software Defined Networking, Wireless Communications and Networking Conference (WCNC), 2017 IEEE, 2017, DOI: $10.1109 /$ WCNC.2017.7925536.

[29] [25] Vudatha, C.P., Nalliboena, S., Jammalamadaka, S.K.R., Duvvuri, B.K.K., Reddy, L.S.S., Automated generation of test cases from output domain of an embedded system using Genetic algorithms, ICECT 2011 - 2011 3rd International Conference on Electronics Computer Technology 5,5941989 , pp. $216-220$

[30] [26] Sastry, J.K.R., Ganesh, J.V., Bhanu, J.S., I2C based networking for implementing heterogeneous microcontroller based distributed embedded systems, Indian Journal of Science and Technology, Volume 8, Issue 15, 2015

[31] [27] Sastry, J.K.R., Naga Sai Tejasvi, T., Aparna, J., Dynamic scheduling of message flow within a distributed embedded system connected through a RS485 network, ARPN Journal of Engineering and Applied Sciences, Volume 12, Issue 9, 1 May 2017, Pages 2809-2817

[32] [28] Sastry, J.K.R., Suresh, A., Bhanu, S.J., Building heterogeneous distributed embedded systems through rs485 communica- tion protocol, ARPN Journal of Engineering and Applied Sciences, 2015, 10(16), pp. 6793-6803

[33] [29] ADITYA VARMA, K.V., MANIDEEP, T. and ASADI, S.S., 2016. A critical comparison of quantity estimation for gated community construction project using Traditional method vs Plan swift software: A case study. International Journal of Civil Engineering and Technology, 7(6), pp. 707-713.

[34] [30] VUNDAVILLI, P.R., PARAPPAGOUDAR, M.B., KODALI, S.P. and BENGULURI, S., 2012. Fuzzy logic-based expert system for prediction of depth of cut in abrasive water jet machining process. Knowledge-Based Systems, 27, pp. 456-464.

[35] [31] KILARU, S., HARIKISHORE, K., SRAVANI, T., ANVESH CHOWDARY, L. and BALAJI, T., 2014. Review and analysis of promising technologies with respect to Fifth generation networks, 1st International Conference on Networks and Soft Computing, ICNSC 2014 - Proceedings 2014, pp. 248-251. 